\title{
Lipopolysaccharide induces inflammation and facilitates lung metastasis in a breast cancer model via the prostaglandin E2-EP2 pathway
}

\author{
SHANCHENG LI, XIAOYA XU, MAN JIANG, YULI BI, JIYING XU and MINGYONG HAN \\ Cancer Therapy and Research Center, Shandong Provincial Hospital, Shandong University, \\ Jinan, Shandong 250021, P.R. China
}

Received February 23, 2014; Accepted November 20, 2014

DOI: $10.3892 / \mathrm{mmr} .2015 .3258$

\begin{abstract}
Inflammation is a potent promoter of tumor metastasis. The aim of the present study was to explore the function of systemic inflammation in the formation of lung metastasis of breast cancer cells in a mouse model. BALB/c mice were injected intraperitoneally with lipopolysaccharide (LPS) in order to establish an inflammatory animal model and 4T1 murine breast cancer cells were injected through the tail vein to induce lung metastasis. The levels of proinflammatory cytokines were evaluated by ELISA. Metastases on the surface of the lungs were counted and histologically analyzed by hematoxylin and eosin staining. Angiogenesis in the lungs was examined by CD31 immunofluorescence. Mouse pulmonary endothelial cells (MPVECs) were isolated and used to assay endothelial tube formation and determine the protein expression levels of vascular endothelial growth factor (VEGF) in vitro. Serum levels of VEGF and prostaglandin E2 (PGE2), the number and size of metastatic lesions, and the expression levels of cyclooxygenase- 2 were significantly greater in the lungs of LPS-treated mice, as compared with those in control mice threated with phosphate-buffered saline. Blood vessel density was also markedly increased in the LPS-treated mice. These increases were reversed by treatment with celecoxib. In vitro, the protein expression levels of VEGF produced by the PGE2-treated cells were significantly increased in a concentration-dependent manner. In addition, the production of VEGF
\end{abstract}

Correspondence to: Professor Mingyong Han, Cancer Therapy and Research Center, Shandong Provincial Hospital, Shandong University, 324 Jingwuweiqi Road, Jinan, Shandong 250021, P.R. China

E-mail: hanmingyong71@126.com

Abbreviations: LPS, lipopolysaccharide; PGE2, prostaglandin E2; VEGF, vascular endothelial growth factor; H\&E, hematoxylin and eosin; MPVECs, mouse pulmonary endothelial cells; PBS, phosphate-buffered solution; OD, optical density

Key words: angiogenesis, breast cancer, lung metastasis, prostaglandin E2-EP2, vascular endothelial growth factor was increased in response to treatment with the PGE2 receptor (EP2) agonist ONO-AE1-259-01; however, this increase was abrogated by treatment with AH6809, an EP2 receptor antagonist. Treatment with PGE2 or VEGF alone promoted the tube formation of MPVECs and this effect was reversed by treatment with celecoxib. These results demonstrated that PGE2 may regulate the release of VEGF by MPVECs through the EP2 receptor pathway and thereby promoted pulmonary angiogenesis and breast cancer metastasis in a mouse model.

\section{Introduction}

Breast cancer is the most common malignancy in females and a critical cause of cancer-associated mortality, second only to lung carcinoma (1). Metastasis, predominantly in the lung, is a leading cause of breast cancer mortality $(2,3)$. Breast cancer metastasis is regulated not only by intrinsic genetic changes in malignant cells, but also by the tumor microenvironment, and emerging data have suggested a role for systemic inflammation in cancer metastasis (3).

Lipopolysaccharide (LPS) is a major structural component of the outer membrane of Gram-negative bacteria and is a potent inducer of inflammation through the production of various cytokines, growth factors and inflammatory mediators (4-7). Previous studies have shown that LPS may induce systemic inflammation and increase hepatic recruitment of cancer cells in mice $(5,8,9)$. In addition, LPS-induced inflammation has been shown to increase the growth of experimental metastases in a murine tumor model and increase angiogenesis in vivo and in vitro (9). These findings were associated with increased vascular endothelial growth factor (VEGF) expression, vascular permeability and tumor cell invasion/migration $(6,10,11)$. Numerous studies have indicated that activation of Toll-like receptor 4 signaling and the nuclear factor- $\kappa \mathrm{B}$ pathway are involved in increases of LPS-induced metastasis in each process, including tumor cell adhesion and invasion $(12,13)$. LPS was previously reported to upregulate the expression of metadherin, which has been shown to induce lung metastasis of 4T1 mammary tumor cells, and has been associated with tumor angiogenesis, including the expression of VEGF and increased microvessel density $(14,15)$. These studies suggested that LPS may promote angiogenesis and 
metastasis. However, the underlying mechanisms remain elusive. The present study used breast cancer cells to generate a metastasis model in LPS-induced mice in order to investigate the underlying mechanisms by which LPS promotes metastasis. Prostaglandin E2 (PGE2) is a metabolite of arachidonic acid derived via the cyclooxygenase pathway. In previous studies, PGE2 was found to play a major role in promoting tumor cell growth and correlate with malignant cell invasion (16). The present study therefore also evaluated the role of PGE2 in breast cancer metastasis.

\section{Materials and methods}

Reagents. Dulbecco's modified Eagle's medium (DMEM), penicillin-streptomycin and $0.25 \%$ trypsin-0.02\% EDTA were obtained from HyClone Laboratories, Inc. (Logan, UT, USA); fetal bovine serum (FBS) was purchased from Gibco Life Technologies (Carlsbad, CA, USA); Matrigel $^{\mathrm{TM}}$ was from BD Biosciences (San Jose, CA, USA); VEGF was from PeproTech, Inc. (Rocky Hill, NJ, USA); and prostaglandin E2 (PGE2), LPS and celecoxib were all purchased from Sigma-Aldrich (St. Louis, MO, USA). AH6809 was obtained from Cayman Chemical Company (Ann Arbor, MI, USA); and the prostaglandin E receptor (EP) agonists (EP1 receptor agonist ONO-DI-004, EP2 receptor agonist ONO-AE1-259-01, EP3 receptor agonist ONO-AE-248 and EP4 receptor agonist ONO-AE1-329) were obtained from Ono Pharmaceutical Co, Ltd. (Osaka, Japan). Monoclonal anti-cyclooxygenase-2 (COX-2; $1: 100)$ and monoclonal $\beta$-actin (1:100) antibodies were purchased from Biosynthesis Biotechnology Co., Ltd. (Beijing, China) and monoclonal anti-VEGF antibody (100 ng/ ml; 1:100; R\&D Systems Inc., Minneapolis, MN, USA); and rat anti-mouse CD31 (1:100) antibody was from Santa Cruz Biotechnology (Dallas, TX, USA).

Animals. A total of 50 female BALB/c mice, aged 6-8 weeks (20-22 g), were obtained from the Animal Center of Shandong University (Jinan, China) and equally assigned to five groups: Phosphate-buffered saline (PBS) group, LPS group, PBS+4T1 group (PT group), LPS+4T1 group (LT group) and LPS+4T1+celecoxib group (LTC group). The mice were housed in five cages with a $12 / 12 \mathrm{~h}$ light-dark cycle at $21-22^{\circ} \mathrm{C}$. Food and water were available ad libitum. Mice were anesthetized with an injection of a 1:1 mixture of Hypnorm (fentanyl citrate, fuanisone; VetaPharma Ltd, Leeds, UK) and Midazolam (dormicum; Roche Diagnostics, Basel, Switzerland) prior to all procedures and sacrificed by a lethal dose of $\mathrm{CO}_{2}$, followed by cervical dislocation. Following sacrification, serum was collected from the mice and stored at $-20^{\circ} \mathrm{C}$ until further use. The lungs were harvested and stored at $-80^{\circ} \mathrm{C}$ or fixed with $4 \%$ formalin. All of the animal experiments were approved by the Institutional Animal Care and Use Committee at the animal facility of Shandong University (Jinan, China).

LPS treatment. The mice in the LPS, LT and LTC groups received intraperitoneal injection of $5 \mathrm{mg} / \mathrm{kg}$ LPS for three consecutive days. This concentration of LPS was sufficiently low to ensure survival of the mice. All of the mice were sacrificed four weeks after the final injection of LPS. The lungs were harvested and systemic inflammation was detected using hematoxylin and eosin (H\&E) staining (Biosynthesis Biotechnology Co., Ltd). Lungs from the various groups of mice were dissected, fixed in $4 \%$ paraformaldehyde (Beijing Biosynthesis Biotechnology Co., Ltd., Beijing, China) for 48h, embedded in paraffin, cut into $3-4-\mu \mathrm{m}$ sections and stained with H\&E.

CD31 immunofluorescence analysis. Mouse lung tissues were cut into 5- $\mu \mathrm{m}$ slices using a YD-355 tissue slicing machine (Jihua Yidi Medical Appliance Co., Ltd, Jinhua, China) and fixed in 3\% paraformaldehyde overnight, followed by treatment with proteinase $\mathrm{K}(20 \mu \mathrm{g} / \mathrm{ml})$. Rat anti-mouse CD31 antibodies were used as primary antibodies (1:50) and goat anti-rat Alex-555 Red (Invitrogen Life Technologies) antibodies were used as the secondary antibodies (1:100). Slides were examined under a confocal microscope (LSM510; Carl Zeiss AG, Oberkochen, Germany). By scanning 10 sections (4-5 $\mu \mathrm{m}$ distances) of each sample, three dimensional images of each tissue sample were assembled.

Experimental lung metastasis model. 4T1 murine breast cancer cells, purchased from the China Cell Culture Centre (Shanghai, China), were resuspended in FBS-free DMEM $\left(1 \times 10^{6}\right.$ cells $\left./ \mathrm{ml}\right)$, and $100 \mu 14 \mathrm{~T} 1$ cells were intravenously injected into each mouse of the PT, LT and LTC groups through the tail vein. Following injection, the mice in the LTC group received treatment with celecoxib $(5 \mathrm{mg} / \mathrm{kg} / \mathrm{d}$ by intraperitoneal injection, once per day) and the other two groups were treated with PBS for 4 weeks until sacrification. Lung metastatic tumors were counted and a pathological examination was performed.

Isolation of endothelial cells. Murine pulmonary endothelial cells (MPVECs) were isolated from the lungs of normal 3-4 week old female BALB/c mice. Once harvested, the lungs were rinsed in PBS and the visceral pleura were stripped. The lungs were minced into $1.0 \times 1.0 \times 1.0 \mathrm{~mm}^{3}$ tissue blocks and cultured in FBS for $30 \mathrm{~min}$. The tissue blocks were then carefully placed in gelatin-coated flasks (Biosynthesis Biotechnology Co., Ltd.) and cultured at $37^{\circ} \mathrm{C}$ in an atmosphere containing $5 \% \mathrm{CO}_{2}$ for $1 \mathrm{~h}$. The flasks were kept inverted to ensure that the tissue blocks were well adhered to the bottom of the culture flasks. Subsequently, the flasks were reverted and DMEM containing 10\% FBS was added. The lung tissue blocks were carefully removed after $60 \mathrm{~h}$ and the culture medium was replaced every other day. Cellular morphology was fusiform or a monolayered 'cobblestone' appearance, which is considered typical for endothelial cells (14).

Endothelial cell culture. MPVECs were grown in $0.1 \%$ gelatin-coated culture flasks with DMEM supplemented with $10 \% \mathrm{FBS}$ and $100 \mathrm{U} / \mathrm{ml}$ penicillin-streptomycin at $37^{\circ} \mathrm{C}$ in a humidified atmosphere containing $5 \% \mathrm{CO}_{2}$. The cells were passaged at a $1: 3$ ratio using $0.25 \%$ trypsin-EDTA once grown to $90 \%$ confluence. Experiments were performed using subcultured cells between the third and sixth in vitro passages. MPVECs were serum-deprived for $2 \mathrm{~h}$ in FBS-free DMEM prior to treatment with various doses of PGE2 $(0,10,100$ and $200 \mathrm{nM}), \mathrm{AH} 6809(10 \mu \mathrm{M})$ and EP receptor agonists $(10 \mu \mathrm{M})$ for $24 \mathrm{~h}$. The culture supernatants were collected following 
centrifugation at $447.6 \mathrm{x}$ g for $15 \mathrm{~min}$ and stored at $-20^{\circ} \mathrm{C}$ until further use.

Cell proliferation assay. The proliferation rate of $4 \mathrm{~T} 1$ cells was determined using an MTT assay. The 4T1 cells were seeded in 96-well plates at a density of $1 \times 10^{5}$ cells/ml and treated with LPS once grown to $90 \%$ confluence. Following incubation with various concentrations of LPS $(0,10,100 \mu \mathrm{g} / \mathrm{ml})$ for $24 \mathrm{~h}$ or with $100 \mu \mathrm{g} / \mathrm{ml}$ LPS for various durations $(0,24,48,96$ and $120 \mathrm{~h}$ ), $20 \mu \mathrm{l}$ MTT (5 mg/ml; HyClone, Logan, UT, USA) was added to the wells and the plates were incubated at $37^{\circ} \mathrm{C}$ for an additional $4 \mathrm{~h}$. The reaction was terminated by lysing the cells with $200 \mu$ dimethyl sulfoxide (HyClone) for $20 \mathrm{~min}$, with agitation. The optical density (OD) of the cells was measured at $590 \mathrm{~nm}$ (ELx800; BioTek Instruments, Inc., Winooski, VT, USA) and the proliferation rate of the 4T1 cells was calculated. All experiments were repeated three times.

Measurement of cytokines by ELISA. VEGF and PGE2 levels in the animal serum samples, and supernatants of the treated monolayer MPVECs were quantified using VEGF and PGE2 ELISA kits (R\&D Systems, Inc.). All experiments were performed in accordance with the manufacturer's instructions. Standards and samples, in duplicate, were added to 96-well plates pre-coated with monoclonal antibody and incubated at room temperature for $2 \mathrm{~h}$. The plates were then washed three times, $100 \mathrm{ng} / \mathrm{ml}$ biotinylated antibody was added and the plates were incubated for another $2 \mathrm{~h}$ at room temperature, followed by incubation with streptavidin-horseradish peroxidase for $20 \mathrm{~min}$. Bound horseradish peroxidase was detected using tetramethylbenzidine, following the final wash with PBS. The reaction was terminated with stop solution and the OD was measured using a Dynatech MR5000 microplate reader at $450 \mathrm{~nm}$ in order to quantify cytokine production.

Western blot analysis. Western blotting was performed to examine the protein expression levels of COX-2 and VEGF in treated MPVECs, as well as in the lungs of treated mice. The cells were treated with various concentrations of PGE2 in six-well plates, washed with PBS and lysed on ice with $80 \mu \mathrm{l} /$ well lysis buffer (HyClone) for $30 \mathrm{~min}$. The lysates were clarified by centrifugation at $15,000 \mathrm{xg}$ for $30 \mathrm{~min}$ at $4^{\circ} \mathrm{C}$. The mouse lungs were lysed with radioimmunoprecipitation assay buffer (HyClone) and the protein samples from each group were collected and quantified using a Bicinchoninic Acid Quantification kit (HyClone), according to the manufacturer's instructions. Equal amounts of protein $(40 \mu \mathrm{g})$ were added to the loading buffer (HyClone), boiled and separated by $10 \%$ SDS-PAGE (BD Biosciences), prior to being electrophoretically transferred to polyvinylidene fluoride membranes. Following blocking with 5\% skimmed milk, the membranes were incubated with anti-COX-2 antibody (1:1,000 dilution) or anti-VEGF antibody (1:300 dilution) overnight at $4{ }^{\circ} \mathrm{C}$, followed by incubation with anti-rabbit secondary antibody for $1 \mathrm{~h}$ at room temperature. The blots were then exposed to enhanced chemiluminescence (ECL) film. The ECL kit, reagents and apparatus were purchased from Biosynthesis Biotechnology Co., Ltd. Chemiluminescence was detected using EpiChemi3 Darkroom imaging system and LabWorks densitometry software, version 4.6 (both from UVP Bioimaging, Upland, CA,
USA). Data were corrected for background signal and normalized to positive controls using RayBio Analysis Tool software (UVP Bioimaging).

MPVEC endothelial tube formation assay. A tube formation assay was used to determine the effects of celecoxib, PGE2 and VEGF on angiogenesis of MPVECs. Matrigel ${ }^{\mathrm{TM}}(80 \mu \mathrm{l})$ was used to coat 24-well plates, which were then incubated at $37^{\circ} \mathrm{C}$ for $30 \mathrm{~min}$, for gelatinization. Serum-deprived MPVECs were resuspended in FBS-free DMEM $\left(1 \times 10^{6}\right.$ cells $\left./ \mathrm{ml}\right)$ and $600 \mu \mathrm{l}$ cell suspension was seeded into each well. Following $1 \mathrm{~h}$ incubation, the MPVECs were treated with VEGF $(100 \mathrm{nM})$ and PGE2 $(100 \mathrm{nM})$, alone or subsequently with celecoxib $(10 \mu \mathrm{M})$. Culture plates were then incubated for $8 \mathrm{~h}$ and images of the tubules and branch points were captured. The number of tubular structures was counted at 10x magnification in five randomly selected fields using a Zeiss Confocal LSM510 microscope (Carl Zeiss AG). The experiments were repeated three times.

Statistical analysis. Values are expressed as the mean \pm standard deviation. One way analysis of variance was followed by the Bonferroni test for post hoc analysis to evaluate statistical significance. Data were analyzed using JMP version 6 (SAS Institute, Cary, NC, USA). $\mathrm{P}<0.05$ was considered to indicate a statistically significant difference.

\section{Results}

LPS promotes lung metastasis of $4 T 1$ cells. To investigate the effects of LPS on lung metastasis, 4T1 cells were intravenously injected into LPS-treated and PBS-treated mice through the tail vein. The mice were sacrificed after 14 days and a significant increase in the incidence of lung metastasis was observed in the mice of the LT group (9/11), as compared with those in the PT group (5/11). Lung metastatic lesions were visualized by H\&E staining. The number and size of metastatic lesions in LPS-treated mice was significantly greater as compared with that in PBS-treated mice (Fig. 1A-D).

\section{LPS induces the production of VEGF and angiogenesis in} lungs. The lungs of the LPS-treated mice were edematous and enlarged as compared with the lungs of those treated with PBS (Fig. 1E and F). The blood vessels in the lungs of LPS-treated mice appeared as primitive and dilated sinusoidal vascular structures, which consisted of disorganized, tortuous and interconnected vascular plexuses (Fig. $1 \mathrm{G}$ and $\mathrm{H}$ ), as determined by CD31 immunofluorescence. Quantification analysis indicated that the total vessel density in the lungs was markedly increased in the LPS-treated mice, as compared with that in the lungs of PBS-treated mice (data not shown). The concentration of VEGF in the serum of the mice was measured by ELISA and was significantly higher in the LPS-treated group compared with that in the PBS-treated group $(214.3 \mathrm{pg} / \mathrm{ml}$ vs. $64.1 \mathrm{pg} / \mathrm{ml})$. Differences between the LPS and PBS groups were statistically significant $(\mathrm{P}<0.05$; Fig. 1I-K).

LPS does not influence the proliferation of 4T1 cells. 4T1 cells were treated with LPS and the proliferation rate was determined using an MTT assay. There was no significant 

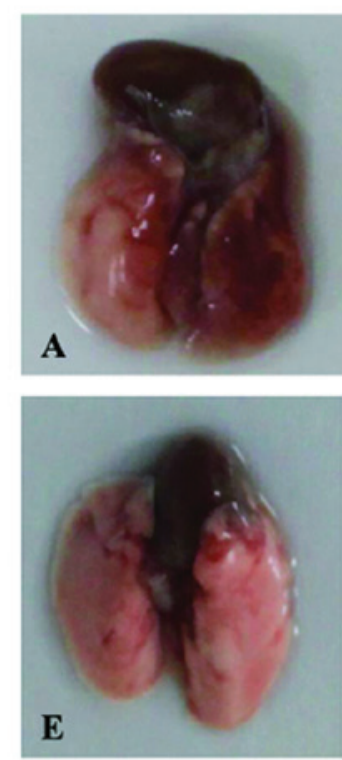

I

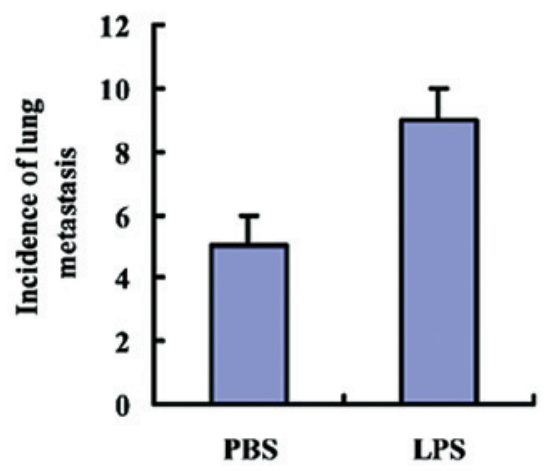

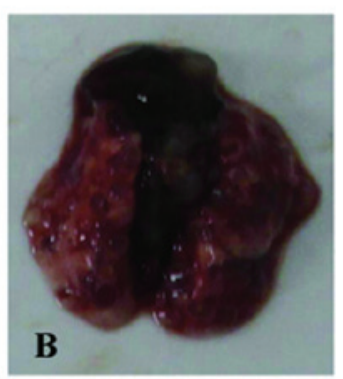

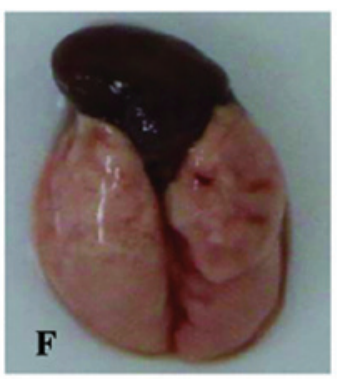

$\mathbf{J}$

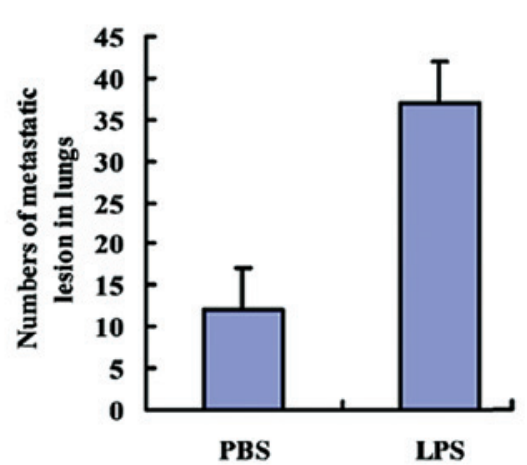

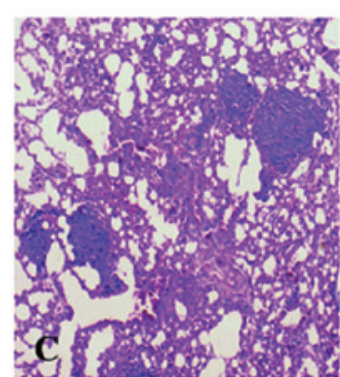
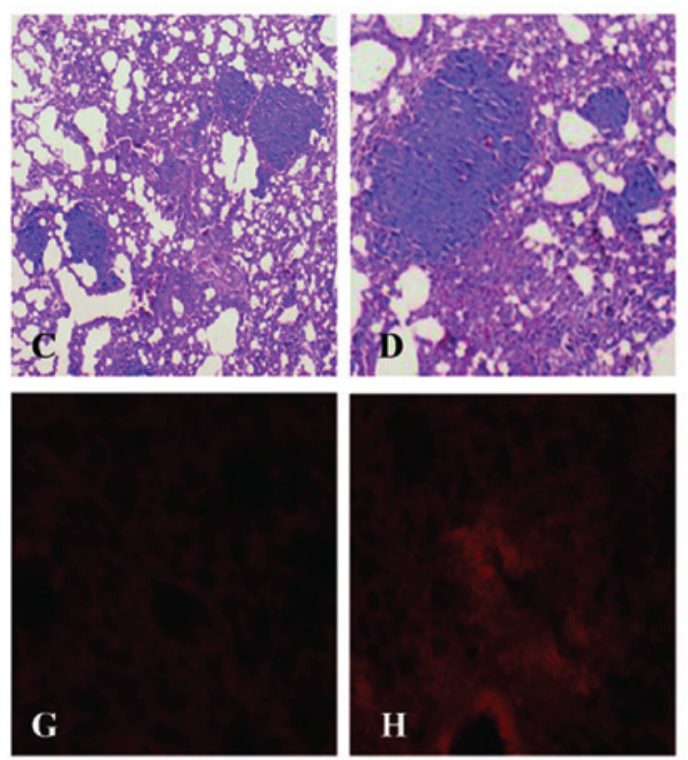

$\mathbf{K}$

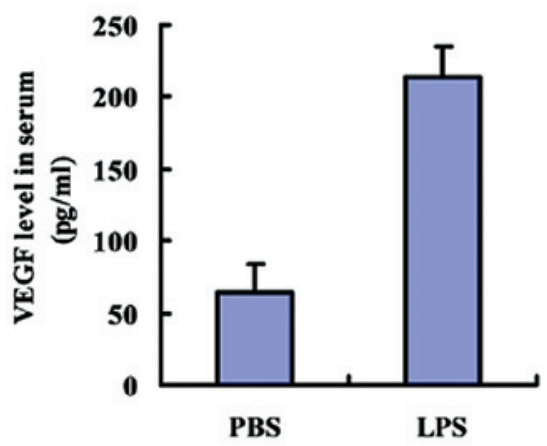

Figure 1. LPS promotes lung metastasis and induces angiogenesis in the lungs of a mouse model. (A) Metastases on the surface of the lungs in the PT group (treated with PBS and 4T1 murine breast cancer cells); (B) metastasis on the surface of the lungs in the LT group (treated with LPS and 4T1); (C) metastatic lesions in the lungs of the PT group (HE; magnification, x40); (D) metastatic lesions in the lungs of the LT group (HE; magnification, $\mathrm{x} 40$ ); (E) lungs of PBS-treated mice; (F) lungs of LPS-treated mice; $(\mathrm{G})$ angiogenesis in the lungs of PBS-treated mice (CD31 immunofluorescence; magnification, $\mathrm{x} 40)$; (H) angiogenesis in the lungs of LPS-treated mice; (I) incidence of lung metastasis $(\mathrm{P}<0.05)$; ( $\mathrm{J})$ number of metastatic lesions in the lungs $(\mathrm{P}<0.05)$; $(\mathrm{K}) \mathrm{VEGF}$ levels (pg/ml) in the serum of PBS-treated and LPS-treated mice $(\mathrm{P}<0.05)$. PT group, treated with PBS+4T1 cells; LT group, treated with LPS+4T1 cells. LPS, lipopolysaccharide; PBS, phosphate-buffered saline; HE, hematoxylin and eosin; VEGF, vascular endothelial growth factor.

A

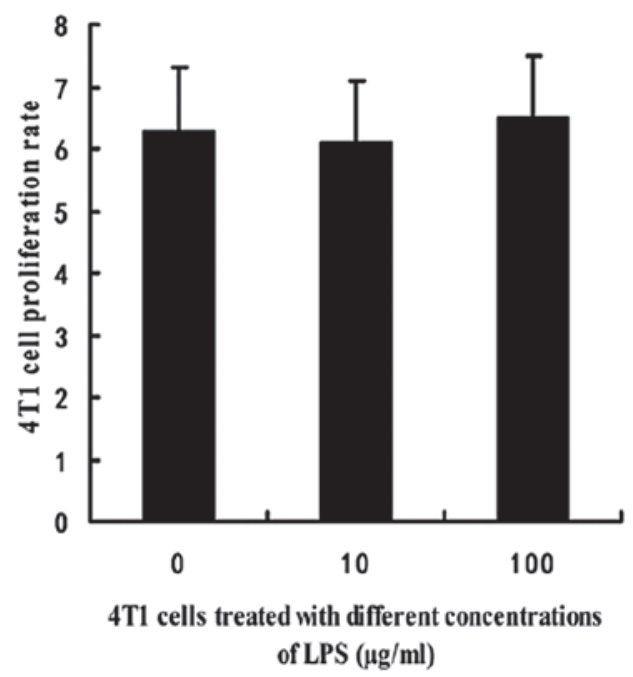

B

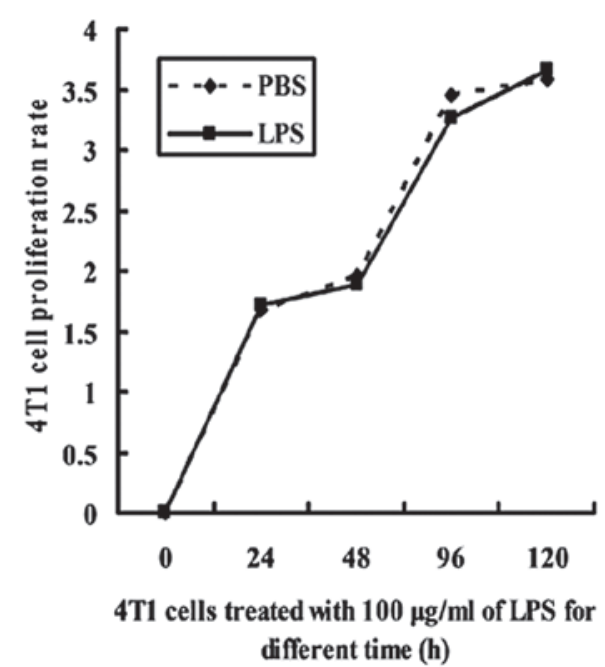

Figure 2. Proliferation of 4T1 murine breast cancer cells, as determined by MTT assay. (A) 4T1 cells treated with various concentrations of LPS ( 0 , 10 and $100 \mu \mathrm{g} / \mathrm{ml})$; (B) 4T1 cells treated with $100 \mu \mathrm{g} / \mathrm{ml}$ LPS for various durations $(0,24,48,96$ and $120 \mathrm{~h})$. PBS, phosphate-buffered saline; LPS, lipopolysaccharide. 

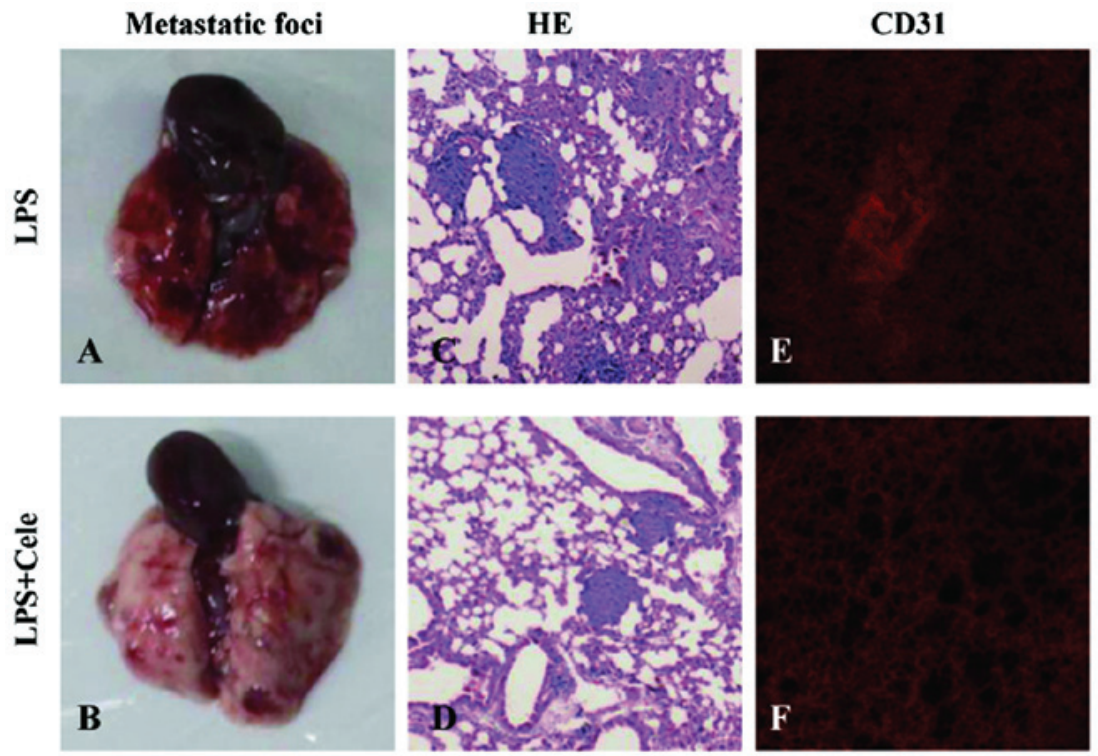

G

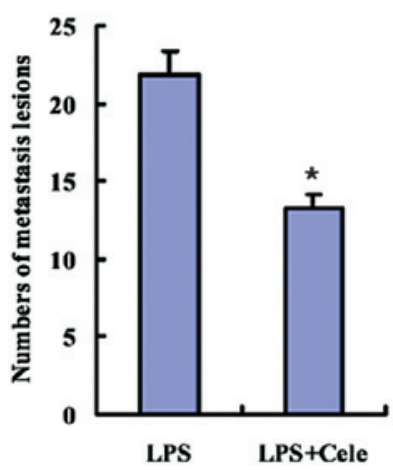

Figure 3. Celecoxib treatment reduces metastasis and angiogenesis in LPS-treated mice. (A) Lung of LT mouse; (B) lung of LTC mouse; (C) metastatic lesions in the lung of LT mice (HE; magnification, $x 40)$; (D) metastatic lesions in the lung of LTC mice (HE; magnification, $\mathrm{x} 40$ ); (E) angiogenesis in LT group (CD31 immunofluorescence; magnification, x40); (F) angiogenesis in LTC group; (G) number of metastatic lesions in the lungs of LPS-treated mice and anti-cyclooxygenase-2 Cele-treated mice $\left({ }^{*} \mathrm{P}<0.05\right)$. LT group, treated with LPS+4T1 cells; LTC group, treated with LPS, 4T1 cells and celecoxib. LPS, lipopolysaccharide; HE, hematoxylin and eosin; Cele, celecoxib.

difference between the proliferative rate of $4 \mathrm{~T} 1$ cells treated with various concentrations of LPS (10 and $100 \mu \mathrm{g} / \mathrm{ml})$ and that of the PBS-treated cells (Fig. 2A). In addition, the proliferative rate of the 4T1 cells treated with $100 \mu \mathrm{g} / \mathrm{ml}$ of LPS for various durations $(0,24,48,96$ and $120 \mathrm{~h})$ was not significantly altered (Fig. 2B).

Celecoxib reduces metastasis and angiogenesis in LPS-treated mice. Anti-COX-2 treatment of the mice in the LT group was performed by intraperitoneal injection of celecoxib $(5 \mathrm{mg} / \mathrm{kg} / \mathrm{d}$, once per day), from the final injection of LPS until the mice were sacrificed. Celecoxib significantly reduced lung metastasis in the tumor-bearing mice (Fig. 3). Laser scanning confocal microscopy detected reduced numbers of CD31-positive cells (red fluorescence) in the lungs of celecoxib-treated LTC mice as compared with those in the mice in the LT group (Fig. 3E and F).

Celecoxib reduces LPS-induced production of VEGF. To verify the effects of PGE2 in systemic inflammation-induced metastasis, the circulating levels of PGE2 and VEGF in the LPS- and celecoxib-treated mice were examined by ELISA. The concentration of PGE2 in the serum of LPS-treated mice was significantly decreased in response to treatment with celecoxib, a selective COX-2 inhibitor. These results were concordant with the levels of VEGF in the serum of LPSand celecoxib-treated mice (Fig. 4A). The release of VEGF by MPVECs into monolayer culture medium was evaluated by ELISA in order to determine whether PGE2 affected the production of VEGF in vitro. Following incubation of the cells with various concentrations of PGE2 (0, 10, 100, $200 \mathrm{nM})$ for $24 \mathrm{~h}$, the production of VEGF was significantly increased in a concentration-dependent manner (Fig. 4C). Furthermore, the production of VEGF was increased almost linearly from the first $(4 \mathrm{~h})$ to the last time-point assessed (48 h) in response to culturing with PGE2 (100 nM) (Fig. 4D). Western blot analysis showed that protein expression levels of VEGF in the PGE2-treated cells were increased in a concentration-dependent manner in PGE2-treated MPVECs as compared with those in the control cells (Fig. 4E).

Treatment with celecoxib inhibits VEGF-induced angiogenesis in vitro. Treatment with PGE2 or VEGF alone promoted tube formation by MPVECs on Matrigel ${ }^{\mathrm{TM}}$, whereas treatment with celecoxib reduced the number of branch points and the total tube length. Treatment with PGE2 also attenuated the celecoxib-induced inhibition of tube formation. However, simultaneous treatment with celecoxib reduced tube formation in the VEGF-treated MPVECs, as compared with that in the control group (Fig. 5).

LPS induces the production of VEGF via the PGE2-EP2 pathway. Four specific EP receptor agonists were used to stimulate MPVECs and the resulting release of VEGF into the monolayer culture medium was quantified by ELISA. Following incubation for $24 \mathrm{~h}$, the EP2 receptor agonist ONO-AE1-259-01 $(10 \mu \mathrm{M})$ significantly increased the production of VEGF, which was significantly alleviated to levels similar to those in the control group in response to treatment with the EP2 receptor antagonist AH6809 (10 $\mu \mathrm{M})$. However, EP1 receptor agonist ONO-DI-004, EP3 receptor agonist ONO-AE-248 and EP4 receptor agonist ONO-AE1-329 had no significant effect on the production of VEGF ( $P>0.05$; Fig. 6).

\section{Discussion}

The results of the present study demonstrated that the incidence of lung metastasis and the number of metastatic lesions was significantly increased in the lungs of LPS-treated mice as compared with those in PBS-treated mice. It may therefore be 
A

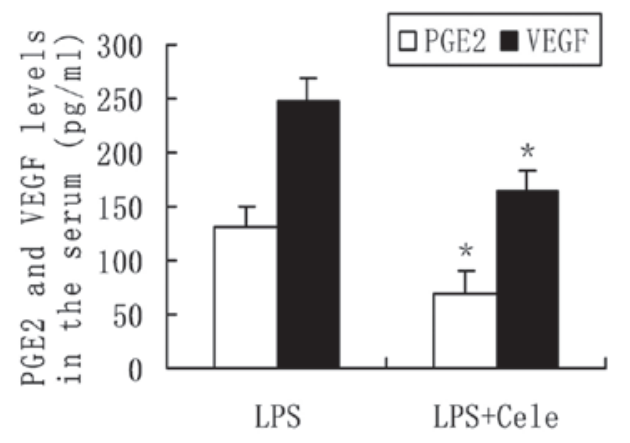

C

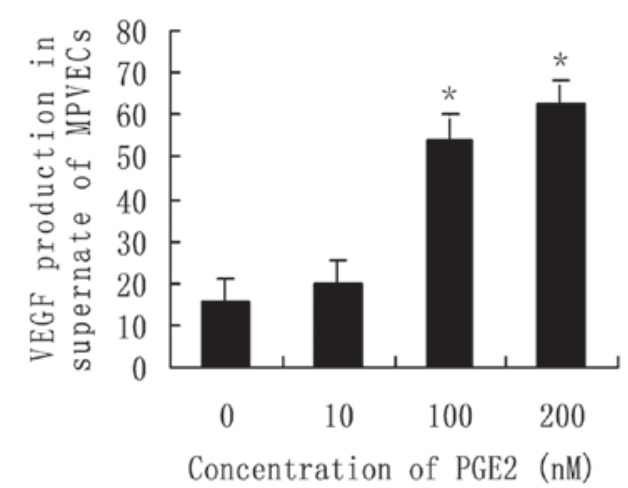

$\mathbf{E}$

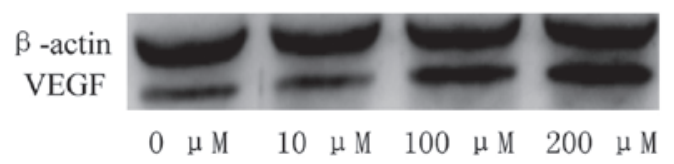

B

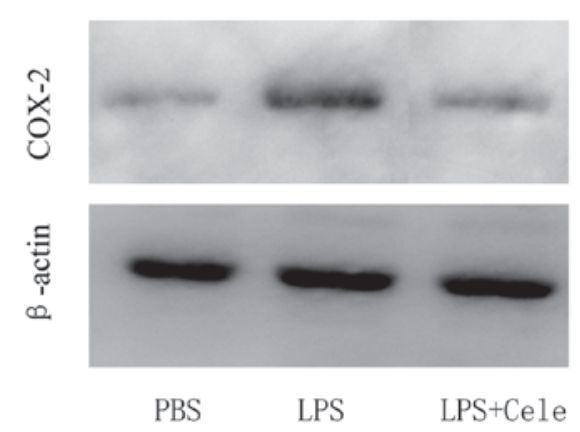

D

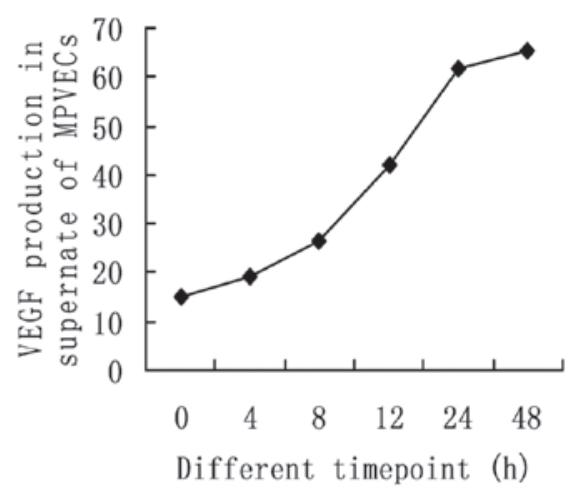

Figure 4. (A) PGE2 and VEGF concentration (pg/ml) in the serum of lipopolysaccharide (LPS)-treated tumor-bearing mice and celecoxib-treated mice ( $\mathrm{P}<0.05)$; (B) COX-2 protein expression levels in the lungs, quantified by western blotting; (C) VEGF production in the supernatant of MPVECs treated with various concentrations of PGE2 (0, 10, 100 and $200 \mathrm{nM})$ for $24 \mathrm{~h}\left({ }^{*} \mathrm{P}<0.05\right)$; (D) VEGF production in the supernatant of MPVECs treated with PGE2 (100 nM) for various durations $(0,4,8,12,24$ and $48 \mathrm{~h})$; (E) VEGF protein expression levels in MPVECs treated with PGE2, as determined by western blotting. PBS, phosphate-buffered saline; PGE2, prostaglandin E2; COX-2, cyclooxygenase-2; MPVECs, murine pulmonary endothelial cells; VEGF, vascular endothelial growth factor.

suggested that inflammation induced by LPS increases metastasis. Compared with the PBS-treated mice, the vessel density in the lungs of the LPS-treated mice was markedly increased, thus suggesting that an inflammatory milieu alongside angiogenesis may be responsible for attracting the 4T1 metastatic cells to the lung microenvironment. Inflammation may instigate cancer initiation and progression through increasing levels of pro-inflammatory mediators, including cytokines, chemokines and transcription factors that mediate tumor cell proliferation, metastasis, invasion and angiogenesis (17-19). To investigate the possible mechanisms by which LPS enhances lung metastasis, the proliferative activity of 4T1 cells was determined, with or without LPS treatment. No significant difference was observed in the rate of cellular proliferation following treatment with LPS at various concentrations and time-points. This excluded the possibility of LPS inducing metastasis through altering the proliferation of cancer cells.

The present study showed that the concentration of VEGF in the serum was markedly increased in LPS-treated mice as compared with that in PBS-treated mice. This suggested that LPS-induced angiogenesis and metastasis in the lungs may be associated with a high expression of VEGF in the inflammatory mouse model. VEGF was initially identified as a vascular permeability factor in the supernatant of a tumor cell line $(20,21)$ and was subsequently reported as a growth factor with mitogenic effects on vascular endothelial cells $(22,23)$. VEGF and its receptors are involved in tumor formation and distant metastasis (24). VEGF promotes endothelial cell survival and regulates migration, permeability and proliferation of blood vessels $(25,26)$, which is critical in tumor angiogenesis and metastasis (27).

PGE2 is a metabolite of arachidonic acid derived from the COX pathway $(28,29)$. Sakurai et al (30) previously reported that activation of COX-2 stimulated angiogenesis in the ovarian corpusluteum, and the effect of PGE2 was shown to overcome the inhibition of angiogenesis by COX-2 inhibitors $(16,31)$. Furthermore, VEGF has been shown to enhance the production of PGE2 by stimulating COX-2 and membrane-associated PGE 

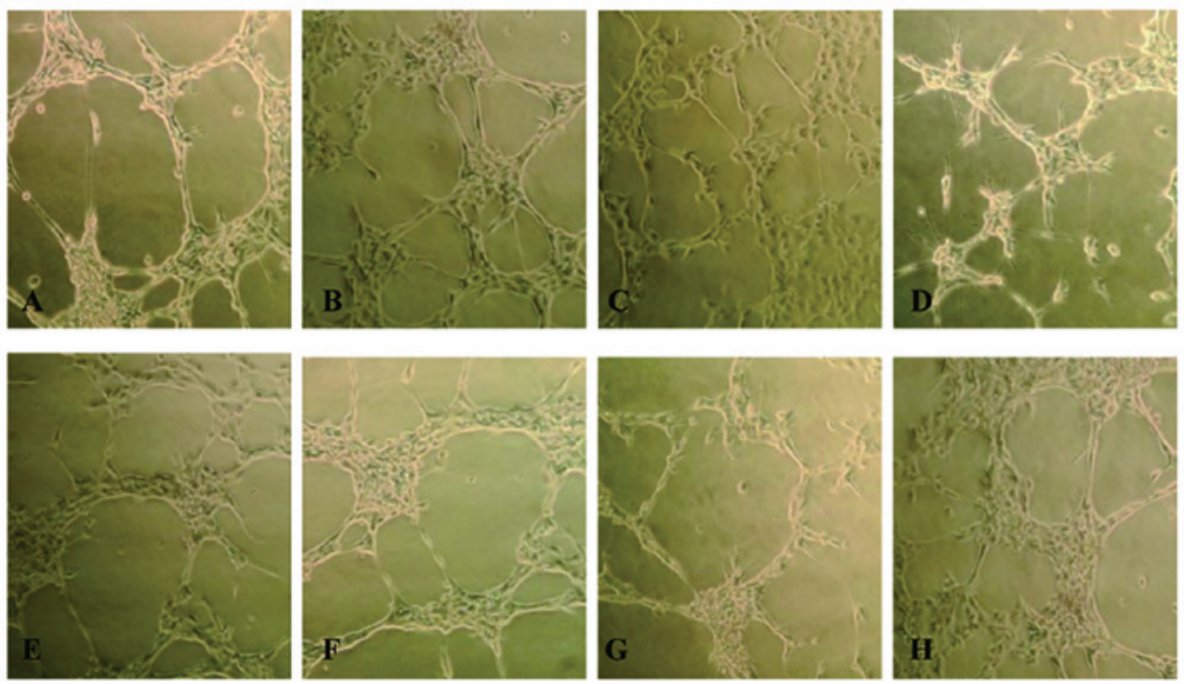

I

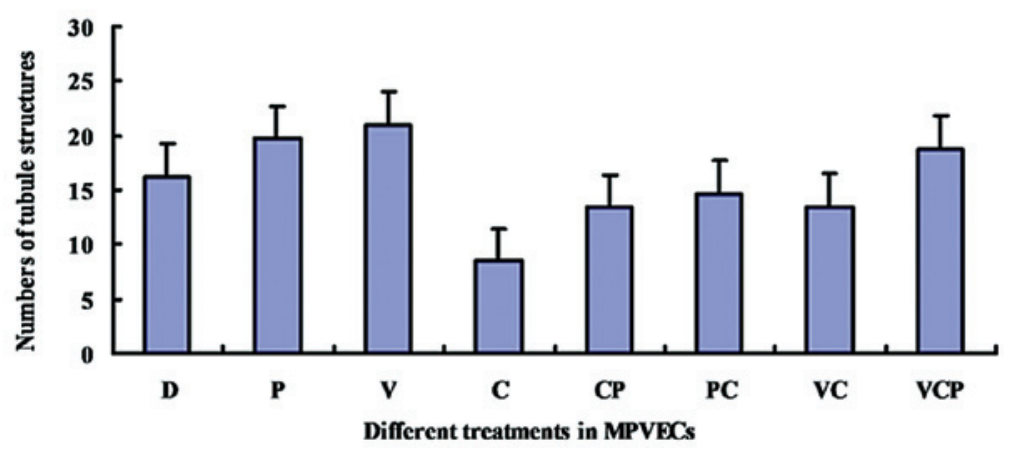

Figure 5. Tube formation assay on BD Matrigel ${ }^{\mathrm{TM}}$. MPVECs were treated VEGF or PGE2 alone or subsequently with celecoxib for $8 \mathrm{~h}$. (A) DMEM; (B) PGE2; (C) VEGF; (D) celecoxib; (E) celecoxib+PGE2; (F) PGE2+celecoxib; (G) VEGF+celecoxib; (H) VEGF+celecoxib+PGE2; (A-H) magnification, x200 (I) Total number of the tubular structures in photographed fields. D, DMEM; P, PGE2; V, VEGF; C, celecoxib. DMEM, Dulbecco's modified Eagle's medium; PGE2, prostaglandin E2; MPVECs, murine pulmonary endothelial cells; VEGF, vascular endothelial growth factor.

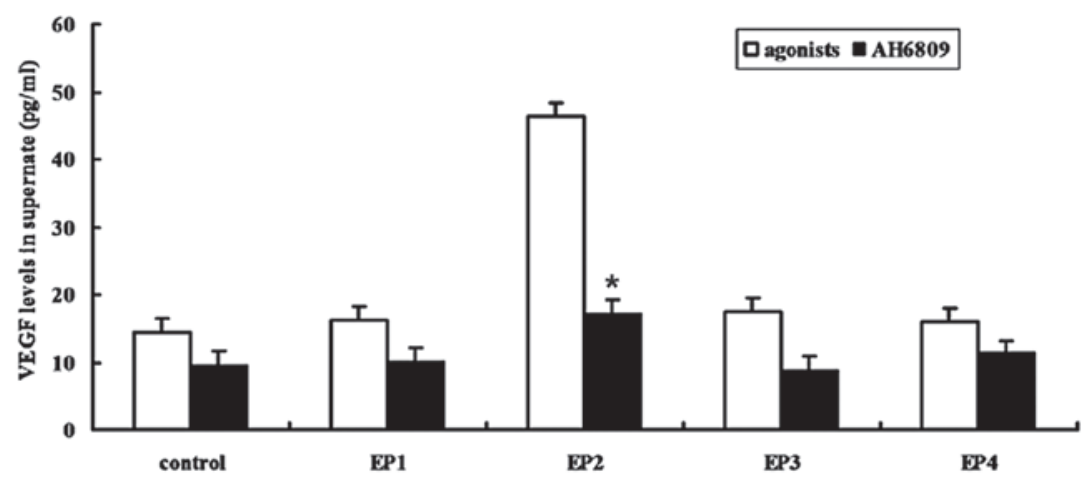

Figure 6. VEGF concentration in monolayer culture medium following treatment of murine pulmonary endothelial cells with four EP receptor agonists and EP2 receptor antagonist AH6809 ("P<0.05). VEGF, vascular endothelial growth factor; EP, prostaglandin E receptor.

synthase-1 expression, and PGE2 concurrently induced VEGF expression in rat ovarian luteal cells (32). Nakanishi et al (27) reported that PGE2 stimulated the release of VEGF from human lung fibroblasts. It was therefore hypothesized that PGE2 may exert a similar effect and serve as a mediator in the release of VEGF by pulmonary endothelial cells. The present study showed that the differences in the serous levels of VEGF between the PBS, LPS and celecoxib-treated groups were concordant with PGE2 levels and COX-2 expression. In vitro, PGE2 treatment increased the production of VEGF in MPVECs in a concentration-dependent manner. Therefore, the results of the present study verified that PGE2 promotes the expression of VEGF in lung endothelial cells.

Angiogenesis is a process whereby new blood vessels grow from existing vessels $(33,34)$, which is an important function of endothelial cells. PGE2 has previously been reported to 
mediate tumor angiogenesis through numerous mechanisms, including increasing the production of VEGF $(35,36)$. In the present study, a tube formation assay was conducted with MPVECs in order to demonstrate the role of PGE2 in tumor angiogenesis. Treatment with PGE2 or VEGF alone promoted tube formation, and these effects were decreased by celecoxib treatment. Furthermore, PGE2 counteracted the celecoxib-induced inhibition of VEGF-stimulated tube formation. These results suggested that VEGF promotes angiogenesis by MPVECs via the PGE2 pathway.

PGE2 exerts its functions through four $\mathrm{G}$ protein-coupled receptors with distinct signaling properties (37). EP1 is associated with Gq protein, and upon stimulation, causes an increase in intracellular $\mathrm{Ca}^{2+}$ and subsequent protein kinase $\mathrm{C}$ activation $(38,39)$. The EP2 and EP4 receptors couple to Gs and protein kinase A/adenyl cyclase and mediate elevations in intracellular cyclic adenosine monophosphate, whereas the EP3 receptor couples to $\mathrm{Gi}$ and decreases concentrations of cyclic adenosine monophosphate (40-42). To identify the specific EP receptor involved in the release of VEGF, the present study treated MPVECs with four EP receptor agonists. Only ONO-AE1-259-01, a specific EP2 receptor agonist, showed an effect similar to that of PGE2, and the specific EP2 receptor antagonist AH6809 counteracted this effect. This indicated that the EP2 receptor participates in regulating the release of VEGF by the PGE2 pathway.

In conclusion, LPS promoted lung metastasis of breast cancer by inducing elevated VEGF production, increased vessel density and angiogenesis of MPVECs via the PGE2 pathway. Therefore, treatment with a PGE2 inhibitor or prostaglandin-EP2 receptor antagonist may provide a novel approach for the prevention and treatment of tumor metastasis.

\section{Acknowledgements}

The present study was supported by the General Programs of Natural Science Foundation of China (grant no. 81272351), the General Programs of Natural Science Foundation of Shandong Province (grant no. ZR2012HM020) and the Key Development Program for Basic Research of Shandong Province (grant no. 2012G0021826).

\section{References}

1. Liu H, Kato Y, Erzinger SA, Kiriakova GM, Qian Y, Palmieri D, Steeg PS and Price JE: The role of MMP-1 in breast cancer growth and metastasis to the brain in a xenograft model. BMC Cancer 12: 583, 2012.

2. Lee YT: Breast carcinoma: pattern of metastasis at autopsy. J Surg Oncol 23: 175-180, 1983.

3. DeSantis C, Siegel R, Bandi P and Jemal A: Breast cancer statistics, 2011. CA Cancer J Clin 61: 409-418, 2011.

4. Harmey JH,Bucana CD,Lu W, Byrne AM, McDonnell S,Lynch C, Bouchier-Hayes D and Dong Z: Lipopolysaccharide-induced metastatic growth is associated with increased angiogenesis, vascular permeability and tumor cell invasion. Int J Cancer 101: 415-422, 2002

5. Ikebe M, Kitaura Y, Nakamura M, Tanaka H, Yamasaki A, Nagai S, Wada J, Yanai K, Koga K, Sato N, Kubo M, Tanaka M, Onishi H and Katano M: Lipopolysaccharide (LPS) increases the invasive ability of pancreatic cancer cells through the TLR4/MyD88 signaling pathway. J Surg Oncol 100: 725-731, 2009.

6. He W, Liu Q, Wang L, Chen W, Li N and Cao X: TLR4 signaling promotes immune escape of human lung cancer cells by inducing immunosuppressive cytokines and apoptosis resistance. Mol Immunol 44: 2850-2859, 2007.
7. Gassmann P, Hemping-Bovenkerk A, Mees ST and Haier J: Metastatic tumor cell arrest in the liver-lumen occlusion and specific adhesion are not exclusive. Int J Colorectal Dis 24: 851-858, 2009.

8. He Z, Zhu Y and Jiang H: Inhibiting toll-like receptor 4 signaling ameliorates pulmonary fibrosis during acute lung injury induced by lipopolysaccharide: an experimental study. Respir Res 10: 126, 2009.

9. Wang EL, Qian ZR, Nakasono M, Tanahashi T, Yoshimoto K, Bando Y, Kudo E, Shimada M and Santo T: High expression of Toll-like receptor 4/myeloid differentiation factor 88 signals correlates with poor prognosis in colorectal cancer. Br J Cancer 102: 908-915, 2010.

10. Yan L, Cai Q and Xu Y: The ubiquitin-CXCR4 axis plays an important role in acute lung infection-enhanced lung tumor metastasis. Clin Cancer Res 19: 4706-4716, 2013.

11. Killeen SD, Wang JH, Andrews EJ and Redmond HP: Bacterial endotoxin enhances colorectal cancer cell adhesion and invasion through TLR-4 and NF-kappaB-dependent activation of the urokinase plasminogen activator system. Br J Cancer 100: 1589-1602, 2009.

12. Liu X, Liang J and Li G: Lipopolysaccharide promotes adhesion and invasion of hepatoma cell lines HepG2 and HepG2.2.15. Mol Biol Rep 37: 2235-2239, 2010.

13. Brown DM and Ruoslahti E: Metadherin, a cell surface protein in breast tumors that mediates lung metastasis. Cancer Cell 5: 365-374, 2004.

14. Zhao Y, Kong X, Li X, Yan S, Yuan C, Hu W and Yang Q: Metadherin mediates lipopolysaccharide-induced migration and invasion of breast cancer cells. PLoS One 6: e29363, 2011.

15. Sethi G, Shanmugam MK, Ramachandran L, Kumar AP and Tergaonkar V: Multifaceted link between cancer and inflammation. Biosci Rep 32: 1-15, 2012.

16. Senger DR, Galli SJ, Dvorak AM, Perruzzi CA, Harvey VS and Dvorak HF: Tumor cells secrete a vascular permeability factor that promotes accumulation of ascites fluid. Science 219: 983-985, 1983.

17. Doan HQ, Bowen KA, Jackson LA and Evers BM: Toll-like receptor 4 activation increases Akt phosphorylation in colon cancer cells. Anticancer Res 29: 2473-2478, 2009.

18. Wang L, Liu Q, Sun Q, Zhang C, Chen T and Cao X: TLR4 signaling in cancer cells promotes chemoattraction of immature dendritic cells via autocrine CCL20. Biochem Biophys Res Commun 366: 852-856, 2008.

19. Ferrara N and Henzel WJ: Pituitary follicular cells secrete a novel heparin-binding growth factor specific for vascular endothelial cells. Biochem Biophys Res Commun1 61: 851-858, 1989.

20. Tsuji T, Aoshiba K, Yokohori N and Nagai A: A systemically administered EP2 receptor agonist stimulates pulmonary angiogenesis in a murine model of emphysema. Prostaglandins Other Lipid Mediat 90: 85-88, 2009.

21. Sato Y, Kanno S, Oda N, Abe M, Ito M, Shitara K and Shibuya M: Properties of two VEGF receptors, Flt-1 and KDR, in signal transduction. Ann NY Acad Sci 902: 201-207, 2000.

22. Lohela M, Bry M, Tammela T and Alitalo K: VEGFs and receptors involved in angiogenesis versus lymphangiogenesis. Curr Opin Cell Biol 21: 154-165, 2009.

23. Perrot-Applanat M and Di Benedetto M: Autocrine functions of VEGF in breast tumor cells: adhesion, survival, migration and invasion. Cell Adh Migr 6: 547-553, 2012.

24. Koch AE: Angiogenesis as a target in rheumatoid arthritis. Ann Rheum Dis 62: ii60-ii67, 2003.

25. Baeriswyl V and Christofori G: The angiogenic switch in carcinogenesis. Semin Cancer Biol 19: 329-337, 2009.

26. Carmeliet P: VEGF as a key mediator of angiogenesis in cancer. Oncology 69 (Suppl 3): 4-10, 2005.

27. Nakanishi M,Sato T,Li Y,Nelson AJ, Farid M, Michalski J, Kanaji N, Wang X, Basma H, Patil A, Goraya J, Liu X, Togo S, L Toews M, Holz O, Muller KC, Magnussen H and Rennard SI: Prostaglandin E2 stimulates the production of vascular endothelial growth factor through the E-prostanoid-2 receptor in cultured human lung fibroblasts. Am J Respir Cell Mol Biol 46: 217-223, 2012.

28. Huang S, Wettlaufer SH, Hogaboam C, Aronoff DM and Peters-Golden M: Prostaglandin $\mathrm{E}_{2}$ inhibits collagen expression and proliferation in patient-derived normal lung fibroblasts via E prostanoid 2 receptor and cAMP signaling. Am J Physiol Lung Cell Mol Physiol 292: L405-L413, 2007.

29. Sakurai T, Tamura K, Okamoto S, Hara T and Kogo H: Possible role of cyclooxygenase II in the acquisition of ovarian luteal function in rodents. Biol Reprod 69: 835-842, 2003. 
30. Sakurai T, Tamura K and Kogo H: Stimulatory effects of eicosanoids on ovarian angiogenesis in early luteal phase in cyclooxygenase-2 inhibitor-treated rats. Eur J Pharmacol 516: $158-164,2005$.

31. Bos CL, Richel DJ, Ritsema T, Peppelenbosch MP and Versteeg HH: Prostanoids and prostanoid receptors in signal transduction. Int J Biochem Cell Biol 36: 1187-1205, 2004.

32. Sakurai T, Tamura K and Kogo H: Vascular endothelial growth factor increases messenger RNAs encoding cyclooxygenase-I and membrane-associated prostaglandin $\mathrm{E}$ synthase in rat luteal cells. J Endocrinol 183: 527-533, 2004.

33. Folkman J and D'Amore PA: Blood vessel formation: what is its molecular basis? Cell 87: 1153-1155, 1996.

34. Keith RL, Geraci MW, Nana-Sinkam SP, Breyer RM, Hudish TM, Meyer AM, Malkinson AM and Dwyer-Nield LD: Prostaglandin E2 receptor subtype 2 (EP2) null mice are protected against murine lung tumorigenesis. Anticancer Res 26: 2857-2861, 2006.

35. Chang SH, Liu CH, Conway R, Han DK, Nithipatikom K, Trifan OC, Lane TF and Hla T: Role of prostaglandin E2-dependent angiogenic switch in cyclooxygenase 2-induced breast cancer progression. Proc Natl Acad Sci USA 101: 591-596, 2004.
36. Gately S and Li WW: Multiple roles of COX-2 in tumor angiogenesis: a target for antiangiogenic therapy. Semin Oncol 31: $2-11,2004$

37. Ma X, Kundu N, Ioffe OB, et al: Prostaglandin E receptor EP1 suppresses breast cancer metastasis and is linked to survival differences and cancer disparities. Mol Cancer Res 8: 1310-1318, 2010.

38. Narumiya S, Sugimoto Y and Ushikubi F: Prostanoid receptors: structures, properties and functions. Physiol Rev 79: 1193-1226, 1999.

39. Yang $\mathrm{T}$ and $\mathrm{Du} Y$ Y: Distinct roles of central and peripheral prostaglandin E2 and EP subtypes in blood pressure regulation. Am J Hypertens 25: 1042-1049, 2012.

40. Sugimoto Y and Narumiya S: Prostaglandin E receptors. J Biol Chem 282: 11613-11617, 2007.

41. Breyer RM, Bagdassarian CK, Myers SA and Breyer MD: Prostanoid receptors: subtypes and signaling. Annu Rev Pharmacol Toxicol 41: 661-690, 2001.

42. Chell S, Kaidi A, Williams AC and Paraskeva C: Mediators of PGE2 synthesis and signalling downstream of COX-2 represent potential targets for the prevention/treatment of colorectal cancer. Biochim Biophys Acta 1766: 104-119, 2006. 